\title{
Analysis of a planar differential system arising from hematology
}

\author{
Lorand Gabriel Parajdi and Radu Precup
}

\begin{abstract}
A complete analysis of a planar dynamic system arising from hematology is provided to confirm the conclusions of computer simulations. Existence and uniqueness for the Cauchy problem, boundedness of solutions and their asymptotic behavior to infinity are established. Particularly, the global asymptotic stability of a steady state is proved in each of the following cases related to leukemia: normal, chronic and accelerated-acute.
\end{abstract}

Mathematics Subject Classification (2010): 34A34, 34D23, 93D20.

Keywords: Nonlinear dynamic system, existence and uniqueness, continuous dependence on data, boundedness, global asymptotic stability, biomathematical model.

\section{Introduction}

We discuss a mathematical model introduced in [5], which describes the cell evolution related to chronic myeloid leukemia. This model is given by the two-dimensional nonlinear differential system

$$
\begin{aligned}
& x^{\prime}(t)=\frac{a x(t)}{1+b_{1} x(t)+b_{2} y(t)}-c x(t) \\
& y^{\prime}(t)=\frac{A y(t)}{1+B(x(t)+y(t))}-C y(t),
\end{aligned}
$$

where $x(t)$ and $y(t)$ represent the normal and abnormal cell populations at time $t$; the model parameters $a$ and $A$ are the nonrestrictive growth rates of normal and abnormal cells; $b_{1}, b_{2}$ and $B$ are the bone marrow microenvironment sensitivities; while $c$ and $C$ stand for their cell death rates. It is assumed that all parameters are positive, $a>c$, $A>C$ and $b_{1} \geq b_{2}$. 
The case when $b_{1}=b_{2}$ was considered by Dingli and Michor [3] (see also [2] and [4]) and was used as a base for a three dimensional model of bone marrow transplantation in [7] and [8].

The steady-states (stationary) solutions of (1.1) and their local asymptotic stability are studied in [5]. Thus, the stationary solutions of (1.1) are

$$
(0,0),(d, 0),(0, D) \text { and }\left(x^{*}, y^{*}\right),
$$

where

$$
d=\frac{1}{b_{1}}\left(\frac{a}{c}-1\right), \quad D=\frac{1}{B}\left(\frac{A}{C}-1\right),
$$

and

$$
x^{*}=\frac{b_{1}}{b_{1}-b_{2}} d-\frac{b_{2}}{b_{1}-b_{2}} D, \quad y^{*}=\frac{b_{1}}{b_{1}-b_{2}}(D-d) .
$$

Note that the equilibrium $\left(x^{*}, y^{*}\right)$ is of biological interest only if its both components are positive, which happens if

$$
b_{1}>b_{2} \text { and } d<D<\left(b_{1} / b_{2}\right) d .
$$

The following result is about the local asymptotic stability of the stationary solutions of system (1.1).

Theorem 1.1. (a) If $D<d$, then $(d, 0)$ is the only steady state which is locally asymptotically stable.

(b) If $b_{1}>b_{2}$ and $d<D<\left(b_{1} / b_{2}\right) d$, then $\left(x^{*}, y^{*}\right)$ is the only steady state which is locally asymptotically stable.

(c) If $D>\left(b_{1} / b_{2}\right) d$, then $(0, D)$ is the only steady state which is locally asymptotically stable.

The above theorem allows us to say that the inequality $D<d$ corresponds to the normal hematopoiesis, the inequality $d<D<\left(b_{1} / b_{2}\right) d$ characterizes the chronic stage of the leukemic disease, while the relation $D>\left(b_{1} / b_{2}\right) d$ stands for the accelerated-acute phase of the disease.

Numerical simulations performed in [5] showed that the solutions $(x, y)$ of $(1.1)$ with positive initial values at $t=0$ are defined, positive and bounded on the whole semiline $\mathbb{R}_{+}$, and that the asymptotic stability of the stationary solutions is in fact global, not only local. However, these facts have not been proved yet. They are the object of the present paper.

\section{Main Results}

\subsection{Existence and Uniqueness}

Theorem 2.1. For any $t_{0} \geq 0$ and $u_{0}=\left(x_{0}, y_{0}\right) \in(0,+\infty)^{2}$, there is a unique saturated solution $u=u\left(\cdot, t_{0}, u_{0}\right)=(x, y)$ of system (1.1) which exists on $\left[t_{0},+\infty\right)$, is of class $C^{\infty}$, with $x>0$ and $y>0$ on $\left[t_{0},+\infty\right)$, and satisfies the initial condition

$$
u\left(t_{0}\right)=u_{0} \text {. }
$$


Proof. The result follows from the global existence and uniqueness of the solution of the Cauchy problem for system (1.1) considered on the open set $\Omega:=(0, \infty)^{2}$, where the right hand sides of the system are functions of class $C^{1}$, hence locally Lipschitz continuous. By the general theory on the Cauchy problem (see. e.g., [1] and [6]), the domain of the saturated solution $u$ is an open interval $\left(t_{-}, t_{+}\right)$with $-\infty \leq t_{-}<t_{0}<t_{+} \leq+\infty$, and any of the limit points of the solution graph as $t \searrow t_{-}$and $t \nearrow t_{+}$is located either at infinity or on the boundary of $\Omega$. Let $\left(t_{+}, u_{+}\right)$ be any limit point, $u_{+}=\left(x_{+}, y_{+}\right)$. Then $u_{+}=\lim _{k \rightarrow \infty} u\left(t_{k}\right)$ for some sequence $\left(t_{k}\right)$ with $t_{k} \nearrow t_{+}$. If the limit point belongs to the boundary of $\Omega$, then $t_{+}, x_{+}$and $y_{+}$are finite and $x_{+}=0$ or $y_{+}=0$. Assume $x_{+}=0$. Using the first equation of the system, we have for $t \in\left[t_{0}, t_{+}\right]$,

$$
x(t)=-\int_{t}^{t_{+}}\left(\frac{a x(s)}{1+b_{1} x(s)+b_{2} y(s)}-c x(s)\right) d s .
$$

By direct computation we can see that

$$
\left|\frac{a}{1+b_{1} x+b_{2} y}-c\right| \leq \max \{a-c, c\}=: \gamma \quad(x, y \geq 0) .
$$

Then

$$
|x(t)| \leq \gamma \int_{t}^{t_{+}}|x(s)| d s \quad\left(t \in\left[t_{0}, t_{+}\right]\right),
$$

whence from Gronwall's inequality, $x(t)=0$ for all $t \in\left[t_{0}, t_{+}\right]$, which is impossible since $x\left(t_{0}\right)=x_{0}>0$. Similarly, the case $y_{+}=0$ is not possible. Hence the limit point $\left(t_{+}, u_{+}\right)$does not belong to the boundary of $\Omega$. Next assume that $t_{+}<+\infty$. Then we must have $x_{+}=+\infty$ or $y_{+}=+\infty$, that is the solution blows up in finite time. If $x_{+}=+\infty$, then we may assume that $x^{\prime}\left(t_{k}\right) \rightarrow+\infty$ too, and using the first equation of the system,

$$
x^{\prime}\left(t_{k}\right)=\frac{a x\left(t_{k}\right)}{1+b_{1} x\left(t_{k}\right)+b_{2} y\left(t_{k}\right)}-c x\left(t_{k}\right) \leq \frac{a x\left(t_{k}\right)}{1+b_{1} x\left(t_{k}\right)}-c x\left(t_{k}\right),
$$

and letting $k \rightarrow+\infty$, we obtain the contradiction $+\infty \leq \frac{a}{b_{1}}-\infty=-\infty$.

Similarly, we can derive a contradiction in case that $y_{+} \stackrel{1}{=}+\infty$. Hence the solution does not blow up in finite time. It remains that $t_{+}=+\infty$, i.e., the solution exists on the whole positive semiline $\left[t_{0},+\infty\right)$. The fact that the solutions are $C^{\infty}$ comes from the $C^{\infty}$ smoothness of the right hand sides of the system.

\subsection{Boundedness of Solutions}

Theorem 2.2. The solution $u=u\left(\cdot, t_{0}, u_{0}\right)$ is bounded on $\left[t_{0},+\infty\right)$ for every $t_{0} \geq 0$ and $u_{0} \in(0,+\infty)^{2}$.

Proof. First we remark that system (1.1) can be rewritten in the form

$$
\begin{aligned}
x^{\prime} & =-b_{2} c x \frac{\alpha x+y-\alpha d}{1+b_{1} x+b_{2} y} \\
y^{\prime} & =-B C y \frac{x+y-D}{1+B(x+y)},
\end{aligned}
$$


where $\alpha=b_{1} / b_{2} \geq 1$. Let $u=(x, y)$. We consider two cases: (a) $D \leq \alpha d$; (b) $D>\alpha d$.

(a) Assume $D \leq \alpha d$. If

$$
x+y-\alpha d \geq 0 \quad \text { on }\left[t_{0},+\infty\right),
$$

then $\alpha x+y-\alpha d \geq 0$ and $x+y-D \geq 0$ on $\left[t_{0},+\infty\right)$. Then $x^{\prime} \leq 0$ and $y^{\prime} \leq$ 0 , that is $x$ and $y$ are decreasing on $\left[t_{0},+\infty\right)$. Being bounded from below by zero, they are bounded functions on $\left[t_{0},+\infty\right)$ as claimed. Assume next that (2.2) does not hold, i.e., there is a point $t_{1} \geq t_{0}$ with $(x+y-\alpha d)\left(t_{1}\right)<0$. We prove that in this case $(x+y-\alpha d)(t) \leq 0$ for all $t \geq t_{4}$ and some $t_{4} \geq t_{1}$, which clearly implies the boundedness of $x$ and $y$. Assume the contrary. Then there are points $t_{3}>t_{2}>t_{1}$ with $(x+y-\alpha d)\left(t_{2}\right)=0$ and

$$
(x+y-\alpha d)(t)>0 \text { on }\left(t_{2}, t_{3}\right) .
$$

Then as above we have $\alpha x+y-\alpha d>0$ and $x+y-D>0$ on $\left(t_{2}, t_{3}\right)$, which guarantees that $x$ and $y$ are strictly decreasing on $\left[t_{2}, t_{3}\right]$. Consequently, we should have $x(t)<x\left(t_{2}\right)$ and $y(t)<y\left(t_{2}\right)$ for all $t \in\left(t_{2}, t_{3}\right)$, whence $(x+y-\alpha d)(t)<$ $(x+y-\alpha d)\left(t_{2}\right)=0$ on $\left(t_{2}, t_{3}\right)$, which is in contradiction with (2.3).

(b) Assume $D>\alpha d$. If

$$
x+y-D \geq 0 \quad \text { on }\left[t_{0},+\infty\right)
$$

then $\alpha x+y-\alpha d \geq 0$ on $\left[t_{0},+\infty\right)$, and so $x^{\prime} \leq 0$ and $y^{\prime} \leq 0$, that is $x$ and $y$ are decreasing on $\left[t_{0},+\infty\right)$. Being bounded from below by zero, they are bounded functions on $\left[t_{0},+\infty\right)$ as claimed. Assume next that (2.4) does not hold, i.e., there is a point $t_{1} \geq t_{0}$ with $(x+y-D)\left(t_{1}\right)<0$. We prove that in this case $(x+y-D)(t) \leq 0$ for all $t \geq t_{4}$ and some $t_{4} \geq t_{1}$, which clearly implies the boundedness of $x$ and $y$. Assuming the contrary, there are points $t_{3}>t_{2}>t_{1}$ with $(x+y-D)\left(t_{2}\right)=0$ and

$$
(x+y-D)(t)>0 \quad \text { on }\left(t_{2}, t_{3}\right) .
$$

Then $\alpha x+y-\alpha d>0$ on $\left(t_{2}, t_{3}\right)$, which guarantees that $x$ and $y$ are strictly decreasing on $\left[t_{2}, t_{3}\right]$, and as above we derive a contradiction.

\subsection{Continuous Dependence on Data}

In practice, it is important to estimate the error between the solution of a given system and the solutions of a perturbed system. Let $u=(x, y)$ be the unique saturated solution of (1.1) satisfying the initial condition $u\left(t_{0}\right)=u_{0}$, where $t_{0} \geq 0$ and $u_{0}=$ $\left(x_{0}, y_{0}\right) \in(0,+\infty)^{2}$, and let $v=(\bar{x}, \bar{y})$ be any solution of a Cauchy problem of the form

$$
\left\{\begin{array}{l}
v^{\prime}=g(t, v) \\
v\left(t_{0}\right)=v_{0},
\end{array}\right.
$$

where $v_{0}=\left(\bar{x}_{0}, \bar{y}_{0}\right) \in \mathbb{R}_{+}^{2}, g=\left(g_{1}, g_{2}\right) \in C\left(\left[t_{0}, t_{0}+h\right] \times \mathbb{R}_{+}^{2} ; \mathbb{R}_{+}^{2}\right)$, and it is assumed that $v$ exists on the interval $\left[t_{0}, t_{0}+h\right]$. 
We are interested to estimate the functions $x-\bar{x}$ and $y-\bar{y}$ in terms of the differences $x_{0}-\bar{x}_{0}, y_{0}-\bar{y}_{0}, f_{1}-g_{1}$ and $f_{2}-g_{2}$, where

$$
\begin{aligned}
& f_{1}(x, y)=\frac{a x}{1+b_{1} x+b_{2} y}-c x \\
& f_{2}(x, y)=\frac{A y}{1+B(x+y)}-C y .
\end{aligned}
$$

By direct computation we can show that $f_{1}, f_{2}$ satisfy the Lipschitz conditions

$$
\begin{aligned}
\left|f_{1}\left(w_{1}, w_{2}\right)-f_{1}\left(\bar{w}_{1}, \bar{w}_{2}\right)\right| & \leq l_{11}\left|w_{1}-\bar{w}_{1}\right|+l_{12}\left|w_{2}-\bar{w}_{2}\right| \\
\left|f_{2}\left(w_{1}, w_{2}\right)-f_{2}\left(\bar{w}_{1}, \bar{w}_{2}\right)\right| & \leq l_{21}\left|w_{1}-\bar{w}_{1}\right|+l_{22}\left|w_{2}-\bar{w}_{2}\right|
\end{aligned}
$$

for $w_{1}, w_{2}, \bar{w}_{1}, \bar{w}_{2} \in \mathbb{R}_{+}$, where

$$
\begin{array}{ll}
l_{11}=\max \{a-c, c\}, & l_{12}=\frac{a b_{2}}{4 b_{1}} \\
l_{21}=\frac{A}{4}, & l_{22}=\max \{A-C, C\} .
\end{array}
$$

Denote

$$
l=\max \left\{l_{11}, l_{12}\right\}+\max \left\{l_{21}, l_{22}\right\} .
$$

Theorem 2.3. Assume that

$$
\begin{aligned}
&\left|f_{1}\left(w_{1}, w_{2}\right)-g_{1}\left(t, w_{1}, w_{2}\right)\right| \leq \eta_{1} \\
&\left|f_{2}\left(w_{1}, w_{2}\right)-g_{2}\left(t, w_{1}, w_{2}\right)\right| \leq \eta_{2}
\end{aligned}
$$

for all $w_{1}, w_{2} \in \mathbb{R}_{+}, t \in\left[t_{0}, t_{0}+h\right]$, and some numbers $\eta_{1}, \eta_{2} \geq 0$. Then

$$
|x(t)-\bar{x}(t)|+|y(t)-\bar{y}(t)| \leq\left(\left|x_{0}-\bar{x}_{0}\right|+\left|y_{0}-\bar{y}_{0}\right|+\left(\eta_{1}+\eta_{2}\right) h\right) e^{h l}
$$

for all $t \in\left[t_{0}, t_{0}+h\right]$.

Proof. The solutions $u=(x, y)$ and $v=(\bar{x}, \bar{y})$ satisfy on the interval $\left[t_{0}, t_{0}+h\right]$ the following integral equations

$$
x(t)=x_{0}+\int_{t_{0}}^{t} f_{1}(x(s), y(s)) d s, \quad y(t)=y_{0}+\int_{t_{0}}^{t} f_{2}(x(s), y(s)) d s
$$

and

$$
\bar{x}(t)=\bar{x}_{0}+\int_{t_{0}}^{t} g_{1}(s, \bar{x}(s), \bar{y}(s)) d s, \quad \bar{y}(t)=\bar{y}_{0}+\int_{t_{0}}^{t} g_{2}(s, \bar{x}(s), \bar{y}(s)) d s .
$$

Subtracting and using (2.7) and (2.8) give

$$
\begin{aligned}
& |x(t)-\bar{x}(t)| \\
\leq & \left|x_{0}-\bar{x}_{0}\right|+\int_{t_{0}}^{t}\left|f_{1}(x(s), y(s))-g_{1}(s, \bar{x}(s), \bar{y}(s))\right| d s \\
\leq & \left|x_{0}-\bar{x}_{0}\right|+\int_{t_{0}}^{t}\left|f_{1}(x(s), y(s))-f_{1}(\bar{x}(s), \bar{y}(s))\right| d s \\
& +\int_{t_{0}}^{t} \mid f_{1}(\bar{x}(s), \bar{y}(s))-g_{1}(s, \bar{x}(s), \bar{y}(s) \mid d s \\
\leq & \left|x_{0}-\bar{x}_{0}\right|+\eta_{1} h+l_{11} \int_{t_{0}}^{t}|x(s)-\bar{x}(s)| d s+l_{12} \int_{t_{0}}^{t}|y(s)-\bar{y}(s)| d s .
\end{aligned}
$$


Hence

$$
\begin{aligned}
& |x(t)-\bar{x}(t)| \\
\leq & \left|x_{0}-\bar{x}_{0}\right|+\eta_{1} h+\max \left\{l_{11}, l_{12}\right\} \int_{t_{0}}^{t}(|x(s)-\bar{x}(s)|+|y(s)-\bar{y}(s)|) d s .
\end{aligned}
$$

Similarly for $|y(t)-\bar{y}(t)|$, we have

$$
\begin{aligned}
& |y(t)-\bar{y}(t)| \\
\leq & \left|y_{0}-\bar{y}_{0}\right|+\eta_{2} h+l_{21} \int_{t_{0}}^{t}|x(s)-\bar{x}(s)| d s+l_{22} \int_{t_{0}}^{t}|y(s)-\bar{y}(s)| d s,
\end{aligned}
$$

whence

$$
\begin{aligned}
& |y(t)-\bar{y}(t)| \\
\leq & \left|y_{0}-\bar{y}_{0}\right|+\eta_{2} h+\max \left\{l_{21}, l_{22}\right\} \int_{t_{0}}^{t}(|x(s)-\bar{x}(s)|+|y(s)-\bar{y}(s)|) d s .
\end{aligned}
$$

Summing up (2.11) and (2.13) gives

$$
\begin{aligned}
|x(t)-\bar{x}(t)|+|y(t)-\bar{y}(t)| \leq & \left|x_{0}-\bar{x}_{0}\right|+\left|y_{0}-\bar{y}_{0}\right|+\left(\eta_{1}+\eta_{2}\right) h \\
& +l \int_{t_{0}}^{t}(|x(s)-\bar{x}(s)|+|y(s)-\bar{y}(s)|) d s .
\end{aligned}
$$

Now Gronwall's inequality yields the result.

Note that estimation (2.9) is given in terms of the norm

$$
\|(x, y)\|=|x|+|y| \text { on } \mathbb{R}^{2} .
$$

With respect to the corresponding norm

$$
\|u\|_{\infty}=\max _{t \in\left[t_{0}, t_{0}+h\right]}\|u(t)\| \text { on } C\left(\left[t_{0}, t_{0}+h\right], \mathbb{R}^{2}\right)
$$

it gives

$$
\|u-v\|_{\infty} \leq\left(\left\|u_{0}-v_{0}\right\|+\|\eta\| h\right) e^{h l} .
$$

Similar estimations hold with respect to other norms on $\mathbb{R}^{2}$. For example, if we consider the norms $\|(x, y)\|=\max \{|x|,|y|\}$ on $\mathbb{R}^{2}$ and the corresponding norm on $C\left(\left[t_{0}, t_{0}+h\right], \mathbb{R}^{2}\right),\|u\|_{\infty}=\max _{t \in\left[t_{0}, t_{0}+h\right]}\|u(t)\|$, we easily obtain (2.14), this time with $l=\max \left\{l_{11}+l_{12}, l_{21}+l_{22}\right\}$. An estimation independent of the norm on $\mathbb{R}^{2}$ can be given in terms of vector-valued norms and matrices.

To this end, let us consider the vector-valued norm on $\mathbb{R}^{2}$,

$$
\|(x, y)\|=(|x|,|y|)^{t r}
$$

and the corresponding vector-valued norm on $C\left(\left[t_{0}, t_{0}+h\right], \mathbb{R}^{2}\right)$,

$$
\|u\|_{\infty}=\left(\|x\|_{\infty},\|y\|_{\infty}\right)^{t r}, \quad u=(x, y) .
$$

If we let $f=\left(f_{1}, f_{2}\right)$, the condition $(2.7)$ can be written in the vector form

$$
\|f(w)-f(\bar{w})\| \leq L\|w-\bar{w}\|
$$


for all $w, \bar{w} \in \mathbb{R}_{+}^{2}$, where $L$ is the square matrix

$$
L=\left[\begin{array}{cc}
\max \{a-c, c\} & \frac{a b_{2}}{4 b_{1}} \\
\frac{A}{4} & \max \{A-C, C\}
\end{array}\right] .
$$

Also condition (2.8) has the vector form

$$
\|f(w)-g(t, w)\| \leq \eta
$$

for all $w \in \mathbb{R}_{+}^{2}, t \in\left[t_{0}, t_{0}+h\right]$, where $\eta=\left(\eta_{1}, \eta_{2}\right)^{t r}$. Furthermore, the inequalities (2.10) and (2.12) can be put together under the vector inequality

$$
\|u(t)-v(t)\| \leq\left\|u_{0}-v_{0}\right\|+h \eta+L \int_{t_{0}}^{t}\|u(s)-v(s)\| d s,
$$

which from the vector version of Gronwall's inequality (see [6], p. 166) gives

$$
\|u(t)-v(t)\| \leq e^{h L}\left(\left\|u_{0}-v_{0}\right\|+h \eta\right),
$$

where $e^{h L}$ is a matrix exponential. Taking the maximum for $t \in\left[t_{0}, t_{0}+h\right]$ finally yields the following conclusion.

Theorem 2.4. Under the above conditions, the following vector inequality holds:

$$
\|u-v\|_{\infty} \leq e^{h L} \gamma
$$

where $\gamma$ is the column vector $\left\|u_{0}-v_{0}\right\|+h \eta$.

\subsection{Global Asymptotic Stability}

Theorem 2.5. For any positive saturated solution $u=(x, y)$ of system (1.1) one has:

$$
\begin{aligned}
& \text { (i) } u(t) \rightarrow(d, 0) \text { as } t \rightarrow+\infty, \text { in case } D<d \text {; } \\
& \text { (ii) } u(t) \rightarrow\left(x^{*}, y^{*}\right) \text { as } t \rightarrow+\infty \text {, in case } d<D<\left(b_{1} / b_{2}\right) d \text {; } \\
& \text { (iii) } u(t) \rightarrow(0, D) \text { as } t \rightarrow+\infty, \text { in case }\left(b_{1} / b_{2}\right) d<D \text {. }
\end{aligned}
$$

Proof. (i) Assume the normal state: $D<d$.

(a) First consider the case where $\alpha x+y-\alpha d \geq 0$ on a positive semiline. Then since $\alpha x+y-\alpha d=\alpha(x+y-d)-(\alpha-1) y$, we also have $x+y-d \geq 0$ and furthermore $x+y-D \geq 0$. Hence both $x$ and $y$ are decreasing on that semiline. Thus their limits $L_{x}, L_{y}$ to $+\infty$ exists, are finite and satisfy

$$
\alpha L_{x}+L_{y}-\alpha d \geq 0
$$

The monotonicity also implies that $x^{\prime}(t) \rightarrow 0$ and $y^{\prime}(t) \rightarrow 0$ as $t \rightarrow+\infty$. Passing to the limit into (2.1) gives

$$
\begin{aligned}
& 0=-b_{2} c \frac{L_{x}\left(\alpha L_{x}+L_{y}-\alpha d\right)}{1+b_{1} L_{x}+b_{2} L_{y}} \\
& 0=-B C \frac{L_{y}\left(L_{x}+L_{y}-D\right)}{1+B\left(L_{x}+L_{y}\right)} .
\end{aligned}
$$

Hence

$$
\begin{aligned}
& L_{x}=0 \text { or } \quad \alpha L_{x}+L_{y}-\alpha d=0 ; \quad \text { and } \\
& L_{y}=0 \text { or } \quad L_{x}+L_{y}-D=0 .
\end{aligned}
$$


The case $L_{x}=L_{y}=0$ is excluded by (2.15). If $L_{x}=0$, then $L_{y}=D$ and from (2.15), $L_{y} \geq \alpha d$, so $D \geq \alpha d$, which is false because $D<d$. Hence $L_{x}>0$. If $L_{y}>0$ too, then from

we obtain

$$
\alpha L_{x}+L_{y}-\alpha d=0, \quad L_{x}+L_{y}-D=0
$$

$$
(1-\alpha) L_{y}=\alpha(d-D)
$$

which is also false since $1-\alpha<0$ and $d-D>0$. It remains that $L_{y}=0$, when from $\alpha L_{x}+L_{y}-\alpha d=0$ we have $L_{x}=d$ and the proof of (i) is finished in this case.

(b) Next assume that $(\alpha x+y-\alpha d)\left(t_{1}\right)<0$ for some $t_{1}$. Then, as in the proof of Theorem 2.2, we can show that $\alpha x+y-\alpha d \leq 0$ on a positive semiline. Then $x$ is increasing on that semiline and being bounded has a finite limit $L_{x}$ and $x^{\prime}(t) \rightarrow 0$ as $t \rightarrow+\infty$. Clearly $L_{x}>0$ (otherwise $x$ would be zero, which is excluded). From the first equation of system (2.1) we deduce that the limit $L_{y}$ of $y(t)$ as $t \rightarrow+\infty$ also exists and

$$
\alpha L_{x}+L_{y}-\alpha d=0
$$

Then for some sequence $\left(t_{n}\right)$ going to $+\infty, y^{\prime}\left(t_{n}\right) \rightarrow 0$ and from the second equation of (2.1),

$$
L_{y}\left(L_{x}+L_{y}-D\right)=0 .
$$

If $L_{y}>0$, then $L_{x}+L_{y}-D=0$ which together with $(2.18)$ implies $(\alpha-1) L_{y}=$ $\alpha(D-d)$ which is false since $\alpha-1>0$ and $D-d<0$. Hence $L_{y}=0$ and consequently $L_{x}=d$ as desired.

(ii) Assume the chronic state: $d<D<\left(b_{1} / b_{2}\right) d$.

In this situation again we are in case (a) from the proof of Theorem 2.2. Since $x, y$ are positive and bounded, the lower and upper limits $\underline{L}_{x}, \underline{L}_{y}, \bar{L}_{x}, \bar{L}_{y}$ of $x$ and $y$ at $+\infty$ are finite. First assume that both limits $\underline{L}_{x}$ and $\underline{L}_{y}$ are strictly positive. We may find a sequence $\left(t_{n}\right)$ with $t_{n} \rightarrow+\infty$ and

$$
x\left(t_{n}\right) \rightarrow \underline{L}_{x}, \quad x^{\prime}\left(t_{n}\right) \rightarrow 0 \text { and } y\left(t_{n}\right) \rightarrow l_{y}
$$

for some number $l_{y}$. Then the first equation in (2.1) yields $\alpha \underline{L}_{x}+l_{y}-\alpha d=0$, whence since $l_{y} \leq \bar{L}_{y}$,

$$
\alpha \underline{L}_{x}+\bar{L}_{y}-\alpha d \geq 0
$$

Similarly, using a sequence $\left(t_{n}\right)$ tending to $+\infty$ and with the properties

$$
y\left(t_{n}\right) \rightarrow \bar{L}_{y}, \quad y^{\prime}\left(t_{n}\right) \rightarrow 0 \text { and } x\left(t_{n}\right) \rightarrow l_{x}
$$

for some number $l_{x}$, from the second equation from (2.1) we deduce $l_{x}+\bar{L}_{y}-D=0$, which in view of $l_{x} \geq \underline{L}_{x}$ yields

$$
\underline{L}_{x}+\bar{L}_{y}-D \leq 0
$$

The last two inequalities imply

$$
(\alpha-1) \underline{L}_{x} \geq \alpha d-D, \quad(\alpha-1) \bar{L}_{y} \leq \alpha(D-d) .
$$

Since

$$
\frac{\alpha d-D}{\alpha-1}=x^{*}, \quad \frac{\alpha(D-d)}{\alpha-1}=y^{*}
$$


we then have

$$
\underline{L}_{x} \geq x^{*}, \quad \bar{L}_{y} \leq y^{*} .
$$

Repeating the same reasoning for the upper limit of $x$ and lower limit of $y$, i.e., using first a sequence $\left(t_{n}\right)$ with $t_{n} \rightarrow+\infty$ and

$$
x\left(t_{n}\right) \rightarrow \bar{L}_{x}, \quad x^{\prime}\left(t_{n}\right) \rightarrow 0 \text { and } y\left(t_{n}\right) \rightarrow l_{y},
$$

and then a sequence satisfying

$$
y\left(t_{n}\right) \rightarrow \underline{L}_{y}, \quad y^{\prime}\left(t_{n}\right) \rightarrow 0 \text { and } x\left(t_{n}\right) \rightarrow l_{x}
$$

we obtain

$$
\alpha \bar{L}_{x}+\underline{L}_{y}-\alpha d \leq 0
$$

and

and finally

$$
\bar{L}_{x}+\underline{L}_{y}-D \geq 0
$$

Consequently

$$
\bar{L}_{x} \leq x^{*}, \quad \underline{L}_{y} \geq y^{*}
$$

$$
\underline{L}_{x}=\bar{L}_{x}=x^{*}, \quad \underline{L}_{y}=\bar{L}_{y}=y^{*},
$$

as wished. Next assume that $0=\underline{L}_{x}$. Then either $x$ approaches 0 at $+\infty$ as a decreasing function on a positive semiline, or $x$ changes monotonicity infinitely many times (oscillates towards infinity). In the first case, when in fact one has $L_{x}=\underline{L}_{x}=\bar{L}_{x}=0$, $x$ is decreasing on a positive semiline and thus $\alpha x+y-\alpha d \geq 0$, and inequality (2.19) immediately follows. In the second case, we may find a sequence $\left(t_{n}\right)$ (of local minima of $x$ ) tending to $+\infty$ with

$$
x^{\prime}\left(t_{n}\right)=0, \quad x\left(t_{n}\right) \rightarrow \underline{L}_{x}=0 \text { and } y\left(t_{n}\right) \rightarrow l_{y} .
$$

Since the values of $x$ and $y$ are strictly positive, hence $x\left(t_{n}\right)>0$, from the first equation in (2.1) we have

$$
\alpha x\left(t_{n}\right)+y\left(t_{n}\right)-\alpha d=0
$$

which implies

$$
\bar{L}_{y}-\alpha d \geq 0
$$

that is inequality (2.19) still holds. Similarly, if $0=\underline{L}_{y}$, inequality (2.20) is still valid.

(iii) Assume the accelerated-acute case: $D>\left(b_{1} / b_{2}\right) d$.

(a) If $x+y \geq D$ on a positive semiline, then $\alpha x+y-\alpha d \geq 0$ too and consequently $x$ and $y$ are decreasing on that semiline. Hence their limits $L_{x}$ and $L_{y}$ at infinity exist, are finite and $x^{\prime}(t), y^{\prime}(t) \rightarrow 0$ as $t \rightarrow+\infty$. Also $L_{x}+L_{y} \geq D$, which makes impossible the case $L_{x}=L_{y}=0$. If $L_{y}=0$, then $L_{x}>0$ and so $\alpha L_{x}+L_{y}-\alpha d=0$. Then $L_{x}=d$ making false the inequality $L_{x}+L_{y} \geq D$. If $L_{x}>0$ and $L_{y}>0$, then from the system $\alpha L_{x}+L_{y}-\alpha d=0, L_{x}+L_{y}-D=0$ we derive $(\alpha-1) L_{x}=\alpha d-D$, which is impossible since the signs of the two sides are opposite. It remains that $L_{x}=0$ and $L_{y}>0$. Then from $L_{x}+L_{y}=D$ we obtain $L_{y}=D$ and we are finished.

(b) If $(x+y-D)\left(t_{1}\right)<0$ for some $t_{1}$, then as above we can prove that $x+y-D \leq 0$ on an whole positive semiline. Then $y$ is increasing on that semiline and being also bounded its limit $L_{y}$ to $+\infty$ exists, is finite and $y^{\prime}(t) \rightarrow 0$ as $t \rightarrow+\infty$. If $L_{y}=0$, 
then $y \equiv 0$ which is excluded from the beginning. Hence $L_{y}>0$. Then from the second equation in (2.1) we find that the limit $L_{x}$ also exists and $L_{x}+L_{y}-D=0$. Then $L_{x}\left(\alpha L_{x}+L_{y}-\alpha d\right)=0$. If $L_{x}>0$, then $\alpha L_{x}+L_{y}-\alpha d=0$ and we derive $(\alpha-1) L_{x}=\alpha d-D$, which is impossible. It remains that $L_{x}=0$, and so $L_{y}=D$ as wished.

Acknowledgement. The authors thank the referee for the remarks that have led to an improved version of the present work.

\section{References}

[1] Barbu, V., Differential Equations, Springer, Cham, 2017.

[2] Cucuianu, A., Precup, R., A hypothetical-mathematical model of acute myeloid leukemia pathogenesis, Comput. Math. Methods Med., 11(2010), 49-65.

[3] Dingli, D., Michor, F., Successful therapy must eradicate cancer stem cells, Stem Cells, 24(2006), 2603-2610.

[4] Parajdi, L.G., Modeling the treatment of tumor cells in a solid tumor, J. Nonlinear Sci. Appl., 7(2014), 188-195.

[5] Parajdi, L.G., Precup, R., Bonci, E.A., A mathematical model of the transition from the normal hematopoiesis to the chronic and accelerated acute stages in myeloid leukemia, submitted.

[6] Precup, R., Ordinary Differential Equations, De Gruyter, Berlin, 2018.

[7] Precup, R., Arghirescu, S., Cucuianu, A., Şerban, M., Mathematical modeling of cell dynamics after allogeneic bone marrow transplantation, Int. J. Biomath., 5(2012), no. 2, Article 1250026, 1-18.

[8] Precup, R., Şerban, M.., Trif, D., Cucuianu, A., A planning algorithm for correction therapies after allogeneic stem cell transplantation, J. Math. Model. Algor., 11(2012), 309-323.

Lorand Gabriel Parajdi

Babeş-Bolyai University, Department of Mathematics

Cluj-Napoca, Romania

e-mail: lorand@cs.ubbcluj.ro

Radu Precup

Babeş-Bolyai University, Department of Mathematics

Cluj-Napoca, Romania

e-mail: r.precup@math . ubbcluj .ro 